\title{
Interpolation inequalities for weak solutions of nonlinear parabolic systems
}

Giuseppe Floridia and Maria Alessandra Ragusa*

\author{
* Correspondence: maragusa@dmi. \\ unict.it \\ Dipartimento di Matematica e \\ Informatica, Universitá di Catania, \\ Viale A. Doria 6, 95125 Catania, \\ Italy
}

\section{Abstract}

The authors investigate differentiability of the solutions of nonlinear parabolic systems of order $2 \mathrm{~m}$ in divergence form of the following type

$$
\sum_{|\alpha| \leq m}(-1)^{|\alpha|} D^{\alpha} a^{\alpha}(X, D u)+\frac{\partial u}{\partial t}=0 .
$$

The achieved results are inspired by the paper of Marino and Maugeri 2008, and the methods there applied.

This note can be viewed as a continuation of the study of regularity properties for solutions of systems started in Ragusa 2002, continued in Ragusa 2003 and Floridia and Ragusa 2012 and also as a generalization of the paper by Capanato and Cannarsa 1981, where regularity properties of the solutions of nonlinear elliptic systems with quadratic growth are reached.

Mathematics Subject Classification (2000)

Primary 35K41, 35K55. Secondary 35B65, 35B45, 35D10

Keywords: Higher order nonlinear parabolic systems, Divergence form, Interpolation theory, Besov spaces, Local differentiability

\section{Introduction}

The study of regularity for solutions of partial differential equations and systems has received considerable attention over the last thirty years. On the other hand, little is known concerning parabolic systems in divergence form of order $2 m$ with quadratic growth and the corresponding analytic properties of solutions. To such classes of systems, our attention is devoted.

This note is a natural continuation of the study, carried out in the last decade and a half, of embedding results of Gagliardo-Nirenberg type from which we deduce local differentiability theorems, making use of interpolation theory in Besov spaces (see e.g. [1-6] and [7]).

In this respect, we mention at first the note [8] where the author proves that, let $\Omega$ $\subset \mathbb{R}^{n}$ an open set, $0<T<\infty$ and $Q=\Omega \times(-T, 0), x^{0}=\left(x_{1}^{0}, x_{2}^{0}, \ldots, x_{n}^{0}\right) \in \Omega \rho>0$ and

$$
\begin{gathered}
B(\rho)=B\left(x^{0}, \rho\right)=\left\{x=\left(x_{1}, x_{2}, \ldots, x_{n}\right):\left|x_{i}-x_{i}^{0}\right|<\rho, i=1,2, \ldots, n\right\}, \text { if } \\
\left.u \in L^{2}\left(-T, 0, H^{1}\left(\Omega, \mathbb{R}^{N}\right)\right) \cap C^{0, \lambda}\left(Q, \mathbb{R}^{N}\right)\right), \quad \forall 0<\lambda<1
\end{gathered}
$$

is a solution of a second order nonlinear parabolic system of variational type and under the assumptions that the coefficients $a^{\alpha}(x, D u)$ have quadratic growth is

\section{Springer}

(C) 2011 Floridia and Ragusa; licensee Springer. This is an Open Access article distributed under the terms of the Creative Commons Attribution License (http://creativecommons.org/licenses/by/2.0), which permits unrestricted use, distribution, and reproduction in any medium, provided the original work is properly cited. 
obtained that

$$
u \in L^{2}\left(-a, 0, H^{1+\theta}\left(B(\sigma), \mathbb{R}^{N}\right)\right)
$$

for every $a \in\left(0, \frac{T}{2}\right), \forall \theta \in(0,1)$ and for each cube $B(2 \sigma) \subset \subset \Omega$.

In the same paper, Fattorusso stressed that it is not possible to improve this result in such a way to achieve, for each solution $u$ to the above system, the differentiability

$$
u \in L^{2}\left(-a, 0, H^{2}\left(B(\sigma), \mathbb{R}^{N}\right)\right)
$$

if, preliminarily, is not ensured the regularity

$$
D_{i} u \in L^{4}\left(-a, 0, L^{4}\left(B(\sigma), \mathbb{R}^{N}\right)\right), \quad i=1, \ldots, n
$$

for every $a \in(0, T)$, and for every $B(2 \sigma) \subset \subset \Omega$,

The technique used in [8] allows the author to achieve, instead of (1.3), the condition

$$
D_{i} u \in L^{2(1+\theta)}\left(-a, 0, L^{4}\left(B(\sigma), \mathbb{R}^{N}\right)\right), \quad i=1, \ldots, n,
$$

for every $a \in(0, T), \forall B(\sigma) \subset \subset \Omega$ and every $\theta \in\left(\frac{n}{n+4 \lambda}, 1\right)$, which is not enough to ensure that is true (1.2)

In [9], under the same assumptions of the previous result [8], the differentiability result (1.2) is proved, for $u$ satisfying (1.1).

Key of this note is the use of interpolation theorems of Gagliardo-Nirenberg type.

The use of interpolation theory, made in [9] and in [1] with montonicity assumption and quadratic growth, as illustrated in [10], has recently allowed Fattorusso and Marino to obtain differentiability also for weak solutions of nonlinear parabolic systems of second order having nonlinearity $1<q<2$ (see for details [11]).

Inspired by the note mentioned above by Marino and Maugeri, in the present note, the authors extend differentiability properties to the case of parabolic systems of order $2 m$. More precisely, let $\Omega$ be an open subset of $\mathbb{R}^{n}, n>2$, and $0<T<\infty$, aim of this note is to study, in the cylinder $Q=\Omega \times(-T, 0)$, the problem of interior local differentiability for solutions

$$
u \in L^{2}\left(-T, 0, H^{m}\left(\Omega, \mathbb{R}^{N}\right)\right) \cap C^{m-1, \lambda}\left(Q, \mathbb{R}^{N}\right), \quad 0<\lambda<1
$$

of the nonlinear parabolic systems of order $2 m$ of variational type

$$
\sum_{|\alpha| \leq m}(-1)^{|\alpha|} D^{\alpha} a^{\alpha}(X, D u)+\frac{\partial u}{\partial t}=0 .
$$

Using the above explained idea is proved the following local differentiability with respect to the spatial derivatives

$$
u \in L^{2}\left(-a, 0, H^{m+1}\left(B(\sigma), \mathbb{R}^{N}\right)\right) \cap H^{1}\left(-a, 0, L^{2}\left(B(\sigma), \mathbb{R}^{N}\right)\right), \quad \forall a \in(0, T), \forall B(\sigma) \subset \subset \Omega .
$$

Let us also mention the considerable note by [1] where the authors prove that a solution $u$ of nonlinear parabolic systems of order 2 with natural growth and coefficients uniformly monotone in $D u$ belongs to

$$
L^{2}\left(-a, 0, H^{2}\left(B(\sigma), \mathbb{R}^{N}\right)\right) \cap H^{1}\left(-a, 0, L^{2}\left(B(\sigma), \mathbb{R}^{N}\right)\right)
$$

Results similar to those obtained by Marino and Maugeri in [9], with stronger assumptions, are obtained by Naumann in [12] and by Naumann and Wolf in [13]. Let 
us also bear in mind the study made by Campanato in [14] on parabolic systems in divergence form.

We want to finish this historical overview, concerning interior differentiability of weak solutions, recalling the recent note [4] where similar results are achieved for elliptic systems of order $2 m$.

\section{Useful assumptions and results}

Let $\Omega$ be an bounded open set in $\mathbb{R}^{n}, n>2, x=\left(x_{1}, x_{2}, \ldots, x_{n}\right)$ denotes a generic point therein, $0<T<\infty$ and $Q$ the cylinder $\Omega \times(-T, 0)$, let $N$ be a positive integer. In $Q$, we consider the following parabolic metric

$$
d(X, Y)=\max \left\{\|x-y\|_{n},|t-\tau|^{\frac{1}{2}}\right\}, \quad X=(x, t), Y=(\gamma, \tau) .
$$

Let us set $k$ a positive integer greater than $1,(\cdot \mid \cdot)_{k}$ and $\|\cdot\|_{k}$ respectively the scalar product and the norm in $\mathbb{R}^{k}$. If there is no ambiguity, we omit the index $k$.

Let $k$ be a nonnegative integer and $\lambda \in] 0,1]$. We denote by $C^{k, \lambda}\left(\bar{Q}, \mathbb{R}^{N}\right)$ the subspace of $C^{k}\left(\bar{Q}, \mathbb{R}^{N}\right)$ of functions $u: \bar{Q} \rightarrow \mathbb{R}^{N}$ that satisfy a Hölder condition of exponent $\lambda$, together with all their derivatives $D^{\alpha} u,|\alpha| \leq k$. If $u \in C^{k, \lambda}\left(\bar{Q}, \mathbb{R}^{N}\right)$, then we set

$$
\|u\|_{C^{k, \lambda}\left(\bar{Q}, \mathbb{R}^{N}\right)}=\sum_{|\alpha| \leq k} \sup _{\bar{Q}}\left\|D^{\alpha} u\right\|_{N}+\sum_{|\alpha|=k}\left[D^{\alpha} u\right]_{\lambda, \bar{Q}}
$$

where

$$
\left[D^{\alpha} u\right]_{\lambda, \bar{Q}}=\sup _{\substack{X, Y \in \bar{Q} \\ X \neq Y}} \frac{\left\|D^{\alpha} u(X)-D^{\alpha} u(Y)\right\|_{N}}{d^{\lambda}(X, Y)}<+\infty, \forall \alpha:|\alpha|=k .
$$

The space $C^{k, \lambda}\left(\bar{Q}, \mathbb{R}^{N}\right)$ is a Banach space, provided with the norm

$$
\|u\|_{C^{k, \lambda}\left(\bar{Q}, \mathbb{R}^{N}\right)}=\|u\|_{C^{k}\left(\bar{Q}, \mathbb{R}^{N}\right)}+\sum_{|\alpha|=k}\left[D^{\alpha} u\right]_{\lambda, \bar{Q}} .
$$

Definition 2.1 (see e.g. $[15,16]$ ). Let $\Omega$ be an bounded open set in $\mathbb{R}^{n}$, let $k$ and $j$ be two positive integers, $k \geq j$. If $p \in\left[1,+\infty\left[\right.\right.$ and $u \in C^{\infty}\left(\bar{\Omega}, \mathbb{R}^{N}\right)$, so we set

$$
|u|_{j, p, \Omega}=\left(\int_{\Omega} \sum_{|\alpha|=j}|| D^{\alpha} u \|_{N}^{p} \mathrm{~d} x\right)^{\frac{1}{p}}, \quad \|\left. u\right|_{k, p, \Omega}=\left(\sum_{j=0}^{k}|u|_{j, p, \Omega}^{p}\right)^{\frac{1}{p}}
$$

and denote respectively by $H^{k, p}\left(\Omega, \mathbb{R}^{N}\right)$ and $H_{0}^{k, p}\left(\Omega, \mathbb{R}^{N}\right)$ the spaces obtained as closure of $C^{\infty}\left(\bar{\Omega}, \mathbb{R}^{N}\right)$ and $C_{0}^{\infty}\left(\Omega, \mathbb{R}^{N}\right)$ regarding the norm $\|u\|_{k, p, \Omega}$. The spaces $H^{k, p}(\Omega$, $\left.\mathbb{R}^{N}\right)$ and $H_{0}^{k, p}\left(\Omega, \mathbb{R}^{N}\right)$ are known in literature as Sobolev Spaces.

We remark that $H^{0, p}\left(\Omega, \mathbb{R}^{N}\right)=L^{p}\left(\Omega, \mathbb{R}^{N}\right), 1 \leq p<+\infty$.

If $p=2$, then we shall simply write $H^{k}\left(\Omega, \mathbb{R}^{N}\right), H_{0}^{k}\left(\Omega, \mathbb{R}^{N}\right),|u|_{j, \Omega},\|u\|_{k, \Omega}$.

Let $\Omega$ be an bounded open set in $\mathbb{R}^{n}$, let us set $\vartheta \in(0,1), p \in[1,+\infty[$.

Definition 2.2. We say that a function $u$ defined in $\Omega$ having values in $\mathbb{R}^{N}$ belongs to $H^{9, p}\left(\Omega, \mathbb{R}^{N}\right)$ if $u \in L^{p}\left(\Omega, \mathbb{R}^{N}\right)$ and is finite 


$$
|u|_{\vartheta, p, \Omega}^{p}=\int_{\Omega} \mathrm{d} x \int_{\Omega} \frac{\|u(x)-u(y)\|_{N}^{p}}{\|x-y\|_{n}^{n+\vartheta p}} \mathrm{~d} y .
$$

Definition 2.3. If $k$ is a nonnegative integer, we mean for $H^{k+\vartheta, p}\left(\Omega, \mathbb{R}^{N}\right)$ the subspace of $H^{k, p}\left(\Omega, \mathbb{R}^{N}\right)$ of functions $u \in H^{k, p}\left(\Omega, \mathbb{R}^{N}\right)$ such that

$$
D^{\alpha} u \in H^{\vartheta, p}\left(\Omega, \mathbb{R}^{N}\right), \quad \forall \alpha:|\alpha|=k .
$$

We stress that $H^{k+\vartheta, p}\left(\Omega, \mathbb{R}^{N}\right)$ is a Banach space equipped with the following norm

$$
\|u\|_{k+\vartheta, p, \Omega}=\left(\|u\|_{k, p, \Omega}^{p}+\sum_{|\alpha|=k}\left|D^{\alpha} u\right|_{\vartheta, p, \Omega}^{p}\right)^{\frac{1}{p}} .
$$

If $p=2$, then we shall simply write $H^{k+9}\left(\Omega, \mathbb{R}^{N}\right)$ and $\|u\|_{k+\vartheta, \Omega}$.

Let $k$ a positive integer, $p \in[1,+\infty[, \vartheta \in(0,1)$, in the following, we will consider the spaces

$$
\begin{aligned}
L^{p}(- & \left.T, 0, H^{k, p}\left(\Omega, \mathbb{R}^{N}\right)\right) \\
& =\left\{u(x, t) \mid u(\cdot, t) \in H^{k, p}\left(\Omega, \mathbb{R}^{N}\right) \text { for a.e. } t \in(-T, 0) \text { and } \int_{-T}^{0}\|u(\cdot, t)\|_{k, p, \Omega}^{p} \mathrm{~d} t<\infty\right\}
\end{aligned}
$$

and

$$
\begin{aligned}
L^{p}( & \left.-T, 0, H^{k+\theta, p}\left(\Omega, \mathbb{R}^{N}\right)\right) \\
& =\left\{u(x, t) \mid u(\cdot, t) \in H^{k+\theta, p}\left(\Omega, \mathbb{R}^{N}\right) \text { for a.e. } t \in(-T, 0) \text { and } \int_{-T}^{0}\|u(\cdot, t)\|_{k+\theta, p, \Omega}^{p} \mathrm{~d} t<\infty\right\} .
\end{aligned}
$$

We say a function $u \in L^{2}\left(-T, 0, H^{m}\left(\Omega, \mathbb{R}^{N}\right) \cap C^{m-1, \lambda}\left(Q, \mathbb{R}^{N}\right), N\right.$ positive integer and 0 $<\lambda<1$, weak solution in $Q$ to the nonlinear parabolic system of order $2 m$

$$
\sum_{|\alpha| \leq m}(-1)^{|\alpha|} D^{\alpha} a^{\alpha}(X, D u)+\frac{\partial u}{\partial t}=0
$$

if

$$
\int_{Q}\left\{\sum_{|\alpha| \leq m}\left(a^{\alpha}(X, D u) \mid D^{\alpha} \varphi\right)-\left(u \mid \frac{\partial \varphi}{\partial t}\right)\right\} \mathrm{d} X=0, \quad \forall \varphi \in C_{0}^{\infty}\left(Q, \mathbb{R}^{N}\right) .
$$

Let us now state some properties useful in the sequel.

Let $\tau \in] 0,1[, \rho$ and $a$ two positive numbers and $h \in \mathbb{R} \backslash\{0\}$, where $|h|<(1-\tau) \rho$.

If $u$ is a function from $B(\rho) \times(-a, 0)$ in $\mathbb{R}^{N}$ and $X=(x, t) \in B(\tau \rho) \times(-a, 0)$, we set

$$
\tau_{i, h} u(X)=u\left(x+h e^{i}, t\right)-u(X), \quad i=1,2, \ldots, n,
$$

where $\left\{e^{i}\right\}_{i=1,2, \ldots, n}$ is the canonic basis of $\mathbb{R}^{n}$.

Let us now state the following results, proved in $[17,18]$ and [19], useful to achieve the main result of the note.

Theorem 2.1. If $u \in L^{p}\left(-a, 0, L^{p}\left(B(2 \rho), \mathbb{R}^{N}\right)\right), a, \rho>0,1<p<+\infty, N$ is a positive integer and exists $M>0$ such that 


$$
\int_{-a}^{0} \mathrm{~d} t \int_{B(\rho)}\left\|\tau_{i, h} u\right\|^{p} \mathrm{~d} x \leq|h|^{p} M, \quad \forall|h|<(1-\tau) \rho, \quad \forall i=1, \ldots, n,
$$

then $u \in L^{p}\left(-a, 0, H^{1, p}\left(B(\rho), \mathbb{R}^{N}\right)\right)$ and

$$
\int_{-a}^{0} \mathrm{~d} t \int_{B(\rho)}\left\|D_{i} u\right\|^{p} \mathrm{~d} x \leq M, \quad \forall i=1, \ldots, n .
$$

Theorem 2.2. Let $u \in H^{1, p}\left(B(\rho), \mathbb{R}^{N}\right)$ for $a, \rho>0,1 \leq p<+\infty$ and $N$ be a positive integer. Then, for every $\tau \in(0,1)$ and every $h \in \mathbb{R},|h|<(1-\tau) \rho$, we have

$$
\left\|\tau_{i, h} u\right\|_{0, p, B(\tau \rho)} \leq|h|\left\|D_{i} u\right\|_{0, p, B(\rho),} \quad i=1,2, \ldots, n .
$$

Theorem 2.3 (see $[18,20]$ ). Let $N$ be a positive integer and $\Omega$ a cube of $\mathbb{R}^{n}$. If

$$
u \in W^{m, r}\left(\Omega, \mathbb{R}^{N}\right) \cap C^{s, \lambda}\left(\Omega, \mathbb{R}^{N}\right),
$$

with $m \geq 2$, $m$ integer, $1<r<\infty, s \geq 0$, s integer, $0<\lambda<1, s<m-1$, then, for each integer $j$ with $s+\lambda<j<m$, there exists two constants $c_{1}$ and $c_{2}$ (depending on $\Omega, m, r$, $s, \lambda, j)$ such that

$$
\max _{|\alpha|=j}\left|D^{\alpha} u\right|_{0, p, \Omega} \leq c_{1}\left(\max _{|\alpha|=m}\left|D^{\alpha} u\right|_{0, r, \Omega}\right)^{\delta} \cdot\left(\max _{|\alpha|=s}\left[D^{\alpha} u\right]_{\lambda, \Omega}\right)^{1-\delta}+c_{2} \max _{|\alpha|=s}\left[D^{\alpha} u\right]_{\lambda, \Omega}
$$

where $\frac{1}{p}=\frac{j}{n}+\delta\left(\frac{1}{r}-\frac{m}{n}\right)-(1-\delta) \frac{s+\lambda}{n}, \forall \delta \in\left[\frac{j-s-\lambda}{m-s-\lambda}, 1[\right.$.

Theorem 2.4 (see [9]). Let $N$ be a positive integer and $\Omega$ a cube of $\mathbb{R}^{n}$. If

$$
u \in W^{m+\theta, r}\left(\Omega, \mathbb{R}^{N}\right) \cap C^{s, \lambda}\left(\Omega, \mathbb{R}^{N}\right),
$$

with $m \geq 1, m$ integer, $0<\theta<1,1<r<\infty, s \geq 0$, s integer, $0<\lambda<1, s<m$, then, for each integer $j$ with $\max \left(s+\lambda, m+\theta-\frac{n}{r}\right)<j<m+\theta$, it results

$$
u \in W^{j, p}\left(\Omega, \mathbb{R}^{N}\right)
$$

and there exists a constant $c$ (depending on $\Omega, m, \theta, r, s, \lambda, j, n, \delta$ ) such that

$$
\|u\|_{j, p, \Omega} \leq c\|u\|_{m+\theta, r, \Omega}^{\delta}\|u\|_{C^{s, \lambda}\left(\Omega, \mathbb{R}^{N}\right)^{\prime}}^{1-\delta}
$$

where $\frac{1}{p}=\frac{j}{n}+\delta\left(\frac{1}{r}-\frac{1 m+\theta}{n}\right)-(1-\delta) \frac{s+\lambda}{n}, \forall \delta \in\left[\frac{j-s-\lambda}{m+\theta-s-\lambda}, 1[\right.$ with $(1-\delta)(s+\lambda)+\delta(m+$ $\theta)$ noninteger.

\section{Interior differentiability of the solutions}

Let us set $m, N$ positive integers, $\alpha=\left(\alpha_{1}, \ldots, \alpha_{n}\right)$ a multi-index and $|\alpha|=\alpha_{1}+\cdots+\alpha_{n}$ the order of $\alpha$. We denote by $\mathcal{R}$ the Cartesian product

$$
\mathcal{R}=\prod_{|\alpha| \leq m} \mathbb{R}_{\alpha}^{N}
$$

and $p=\left\{p^{\alpha}\right\}_{|\alpha| \leq m}, p^{\alpha} \in \mathbb{R}^{N}$, the generic point of $\mathcal{R}$. If $p \in \mathcal{R}$, we set $p=\left(p^{\prime}, p^{\prime \prime}\right)$ where $p^{\prime \prime}=\left\{p^{\alpha}\right\}_{|\alpha|=m} \in \mathcal{R}^{\prime \prime}=\prod_{|\alpha|=m} \mathbb{R}_{\alpha}^{N}, p^{\prime \prime}=\left\{p^{\alpha}\right\}_{|\alpha|=m} \in \mathcal{R}^{\prime \prime}=\prod_{|\alpha|=m} \mathbb{R}_{\alpha}^{N}$, and 


$$
\|p\|^{2}=\sum_{|\alpha| \leq m}\left\|p^{\alpha}\right\|_{N^{\prime}}^{2} \quad\left\|p^{\prime}\right\|^{2}=\sum_{|\alpha|<m}\left\|p^{\alpha}\right\|_{N^{\prime}}^{2} \quad\left\|p^{\prime \prime}\right\|^{2}=\sum_{|\alpha|=m}\left\|p^{\alpha}\right\|_{N}^{2} .
$$

We consider, as usual,

$$
\begin{aligned}
D_{i} & =\frac{\partial}{\partial x_{i}}, i=1, \ldots, n ; \quad D^{\alpha}=D_{1}^{\alpha_{1}} D_{2}^{\alpha_{2}} \ldots D_{n}^{\alpha_{n}} ; \\
D u & =\left\{D^{\alpha} u\right\}_{|\alpha| \leq m}, \quad D^{\prime} u=\left\{D^{\alpha} u\right\}_{|\alpha|<m}, \quad D^{\prime \prime} u=\left\{D^{\alpha} u\right\}_{|\alpha|=m} .
\end{aligned}
$$

Let us consider the following differential nonlinear variational parabolic system of order $2 m$ :

$$
\sum_{|\alpha| \leq m}(-1)^{|\alpha|} D^{\alpha} a^{\alpha}(X, D u)+\frac{\partial u}{\partial t}=0
$$

where $a^{\alpha}(X, p)=a^{\alpha}\left(X, p^{\prime}, p^{\prime \prime}\right)$ are functions of $\Lambda=Q \times \mathcal{R}$ in $\mathbb{R}^{N}$, satisfying the following conditions:

(3.2) for every $\alpha:|\alpha|<m$ and every $p \in \mathcal{R}$, the function $X \rightarrow a^{\alpha}(X, p)$, defined in $Q$ having values in $\mathbb{R}^{N}$, is measurable in $X$;

(3.3) for every $\alpha:|\alpha|<m$ and every $X \in Q$, the function $p \rightarrow a^{\alpha}(X, p)$, defined in Rhaving values in $\mathbb{R}^{N}$, is continuous in $p$;

(3.4) for every $\alpha:|\alpha|<m$ and every $(X, p) \in \Lambda$, such that $\left\|p^{\prime}\right\| \leq K$, we have

$$
\left\|a^{\alpha}(X, p)\right\| \leq M(K)\left(\left|f^{\alpha}(X)\right|+\left\|p^{\prime \prime}\right\|^{2}\right),
$$

where $f^{\alpha} \in L^{2}(Q)$;

(3.5) for every $\alpha:|\alpha|=m$, the function $a^{\alpha}\left(X, p^{\prime}, p^{\prime \prime}\right)$, defined in $Q \times \mathcal{R}$ having values in $\mathbb{R}^{N}$, are of class $C^{1}$ in $Q \times \mathcal{R}$ and, for every $\left(X, p^{\prime}, p^{\prime \prime}\right) \in Q \times \mathcal{R}$ with $\left\|p^{\prime}\right\| \leq K$, we have

$$
\begin{aligned}
\left\|a^{\alpha}\right\|+ & \sum_{r=1}^{n}\left\|\frac{\partial a^{\alpha}}{\partial x_{r}}\right\|+\sum_{k=1}^{N} \sum_{|\beta|<m}\left\|\frac{\partial a^{\alpha}}{\partial p_{k}^{\beta}}\right\| \leq M(K)\left(1+\left\|p^{\prime \prime}\right\|\right), \\
& \sum_{k=1}^{N} \sum_{|\beta|=m}\left\|\frac{\partial a^{\alpha}}{\partial p_{k}^{\beta}}\right\| \leq M(K) ;
\end{aligned}
$$

(3.6) $\exists v=v(K)>0$ such that:

$$
\sum_{h, k=1}^{N} \sum_{|\alpha|=|\beta|=m} \frac{\partial a_{h}^{\alpha}(X, p)}{\partial p_{k}^{\beta}} \xi_{h}^{\alpha} \xi_{k}^{\beta} \geq v(K) \sum_{|\beta|=m}\left\|\xi^{\beta}\right\|_{N}^{2}=v\|\xi\|^{2},
$$

for every $\xi=\left(\xi^{\alpha}\right) \in R^{\prime \prime}$ and for every $(X, p) \in Q \times \mathcal{R}$, with $\left\|p^{\prime}\right\| \leq K$. If the coefficients $a^{\alpha}$ satisfy condition (3.6) we say that the system (3.1) is strictly elliptic in $\Omega$.

Theorem 3.1. If $u \in L^{2}\left(-T, 0, H^{m}\left(\Omega, \mathbb{R}^{N}\right)\right) \cap C^{m-1, \lambda}\left(Q, \mathbb{R}^{N}\right), 0<\lambda<1$, is a weak solution of the system (3.1) and if the assumptions (3.2)-(3.6) hold, then $\forall B(3 \sigma)=B\left(x^{0}, 3 \sigma\right)$ $\subset \subset \Omega, \forall a, b \in(0, T), a<b$, it results

$$
u \in L^{4}\left(-a, 0, H^{m, 4}\left(B(\sigma), \mathbb{R}^{N}\right)\right)
$$


and the following estimate holds

$$
\int_{-a}^{0}\|u\|_{m, 4, B(\sigma)}^{4} d t \leq c(v, K, U, \lambda, \sigma, a, b, m, n)\left\{1+\sum_{|\alpha|<m}\left(\int_{-b}^{0} \mid f^{\alpha} \|_{0, B(3 \sigma)} \mathrm{d} t\right)^{\frac{1+\vartheta}{2}}+\int_{-b}^{0}|u|_{m, B(3 \sigma)}^{2} \mathrm{~d} t\right\}
$$

where $K=\sup _{Q}\left\|D^{\prime} u\right\|$ and $U=\|u\|_{C^{m-1, \lambda}\left(\bar{Q}, \mathbb{R}^{N}\right)}$.

Proof Let us observe that, using Theorem 2.III in [21], for every $0<\vartheta<1$ and $b^{*}=\frac{a+b}{2}$, we have

$$
u \in L^{2}\left(-b^{*}, 0, H^{m+\vartheta}\left(B\left(\frac{5}{2} \sigma\right), \mathbb{R}^{N}\right)\right),
$$

and

$$
\begin{aligned}
& \int_{-b^{*}}^{0}\left|D^{\prime \prime} u\right|_{\vartheta, B\left(\frac{5}{2} \sigma\right)}^{2} \mathrm{~d} t \\
& \leq c(\nu, K, U, \vartheta, \lambda, \sigma, a, b, m, n)\left\{1+\sum_{|\alpha|<m}\left(\int_{-b}^{0}|| f^{\alpha} \|_{0, B(3 \sigma)} \mathrm{d} t\right)^{\frac{1+\vartheta}{2}}+\int_{-b^{*}}^{0}|u|_{m, B(3 \sigma)}^{2} \mathrm{~d} t\right\} .
\end{aligned}
$$

Hence, we remark that $u \in C^{m-1, \lambda}\left(\bar{\Omega}, \mathbb{R}^{N}\right)$, then, it results, for $a . e . t \in\left(-b^{*}, 0\right)$,

$$
u(x, t) \in H^{m+\vartheta}\left(B\left(\frac{5}{2} \sigma\right), \mathbb{R}^{N}\right) \cap C^{m-1, \lambda}\left(\overline{B\left(\frac{5}{2} \sigma\right)}, \mathbb{R}^{N}\right), \quad \forall 0<\vartheta<1, \quad \forall B(3 \sigma) \subset \subset \Omega .
$$

Then, from Theorem 2.4 with $\Omega=B\left(\frac{5}{2} \sigma\right), 1-\lambda<\theta<1$, for $\delta=\frac{1}{2}$, and for a.e. $t \in$ $\left(-b^{*}, 0\right)$ :

$$
u(x, t) \in H^{m, p}\left(B\left(\frac{5}{2} \sigma\right), \mathbb{R}^{N}\right)
$$

and there exists a constant $c=c(\theta, \lambda, \sigma, m, n)$ such that

$$
\|u\|_{m, p, B\left(\frac{5}{2} \sigma\right)} \leq c\|u\|_{m+\theta, B\left(\frac{5}{2} \sigma\right)}^{\frac{1}{2}}\|u\|_{C^{m-1, \lambda}\left(B\left(\frac{5}{2} \sigma\right), \mathbb{R}^{N}\right)^{\prime}}^{\frac{1}{2}}
$$

where $p=4+\frac{8(\theta+\lambda-1)}{n-2(\theta+\lambda-1)}>4$.

The choice $\theta=1-\frac{\lambda}{2}(>1-\lambda)$ ensures that for a. e. $t \in\left(-b^{*}, 0\right)$ we have

$$
u(x, t) \in H^{m, p}\left(B\left(\frac{5}{2} \sigma\right), \mathbb{R}^{N}\right), \quad \text { with } p=4+\frac{4 \lambda}{n-\lambda}, \quad \forall B(3 \sigma) \subset \subset \Omega .
$$

and

$$
\|u\|_{m, p, B\left(\frac{5}{2} \sigma\right)} \leq c(\theta, \lambda, \sigma, m, n)\|u\|_{m+1-\frac{\lambda}{2}, B\left(\frac{5}{2} \sigma\right)}^{\frac{1}{2}}\|u\|_{C^{m-1, \lambda}\left(B\left(\frac{5}{2} \sigma\right), \mathbb{R}^{N}\right)^{\prime}}^{\frac{1}{2}}
$$

where $p=4+\frac{4 \lambda}{n-\lambda}>4$. 
Then we have, for a. e. $t \in\left(-b^{*}, 0\right)$, the following inclusion between Sobolev spaces

$$
u(x, t) \in H^{m, p}\left(B\left(\frac{5}{2} \sigma\right), \mathbb{R}^{N}\right) \subset \subset H^{m, 4}\left(B\left(\frac{5}{2} \sigma\right), \mathbb{R}^{N}\right)
$$

then, using (3.9), written with $\theta=1-\frac{\lambda}{2}$, and (3.10)-(3.12), we have

$$
\begin{aligned}
& \int_{-b^{*}}^{0}\|u\|_{m, 4, B\left(\frac{5}{2} \sigma\right)}^{4} \mathrm{~d} t \leq c(\sigma) \int_{-b^{*}}^{0}\|u\|_{m, 4+\frac{4 \lambda}{n-\lambda}, B\left(\frac{5}{2} \sigma\right)}^{4} \mathrm{~d} t \\
& \leq c(\theta, \lambda, \sigma, m, n) \int_{-b^{*}}^{0}\left\|D^{\prime \prime} u\right\|_{1-\frac{\lambda}{2}, B\left(\frac{5}{2} \sigma\right)}^{2}\|u\|_{C^{m-1, \lambda}\left(B\left(\frac{5}{2} \sigma\right), \mathbb{R}^{N}\right)}^{2} \mathrm{~d} t \\
& \leq c(\nu, K, U, \lambda, \sigma, m, n)\left\{1+\sum_{|\alpha|<m}\left(\int_{-b}^{0}\left\|f^{\alpha}\right\|_{0, B(3 \sigma)} \mathrm{d} t\right)^{\frac{1+\vartheta}{2}}+\int_{-b}^{0}|u|_{m, B(3 \sigma)}^{2} \mathrm{~d} t\right\},
\end{aligned}
$$

then it follows the requested inequality (3.8).

Theorem 3.2 (main result). If $u \in L^{2}\left(-T, 0, H^{m}\left(\Omega, \mathbb{R}^{N}\right)\right) \cap C^{m-1, \lambda}\left(Q, \mathbb{R}^{N}\right), 0<\lambda<1$, is $a$ weak solution of the system (3.1) and if the assumptions (3.2)-(3.6) hold, then $\forall B(3 \sigma)$ $=B\left(x^{0}, 3 \sigma\right) \subset \subset \Omega, \forall a, b \in(0, T), a<b$ it results

$$
u \in L^{2}\left(-a, 0, H^{m+1}\left(B(\sigma), \mathbb{R}^{N}\right)\right) \cap H^{1}\left(-a, 0, L^{2}\left(B(\sigma), \mathbb{R}^{N}\right)\right)
$$

and the following estimate holds

$$
\begin{aligned}
& \int_{-a}^{0}\left\{|u|_{m+1, B(\sigma)}^{2}+\left|\frac{\partial u}{\partial t}\right|_{0, B(\sigma)}^{2}\right\} \mathrm{d} t \\
& \leq c(\nu, K, U, \lambda, \sigma, a, b, m, n)\left\{1+\sum_{|\alpha|<m}\left(\int_{-b}^{0}|| f^{\alpha} \|_{0, B(3 \sigma)} \mathrm{d} t\right)^{2}+\int_{-b}^{0}|u|_{m, B(3 \sigma)}^{2} \mathrm{~d} t\right\}^{(3.15}
\end{aligned}
$$

where $K=\sup _{Q}\left\|D^{\prime} u\right\|$ and $U=\|u\|_{C^{m-1, \lambda}\left(\bar{Q}, \mathbb{R}^{N}\right)}$.

Proof Let us fix $B(3 \sigma)=B\left(x^{0}, 3 \sigma\right) \subset \subset \Omega, a, b \in(0, T)$ with $a<b$ and $h \in \mathbb{R}$ such that $|h|<\frac{\sigma}{2}$, set $b^{*}=\frac{a+b}{2}$, and let $\psi(x) \in C_{0}^{\infty}\left(\mathbb{R}^{n}\right)$ a real function satisfying the following properties $0 \leq \psi \leq 1$ in $\mathbb{R}^{n}, \psi=1$ in $B(\sigma), \psi=0$ in $\mathbb{R}^{n} \mid B(2 \sigma),\|D \psi\| \leq \frac{c}{\sigma}$ in $\mathbb{R}^{n}$.

Let us also define the function $\rho_{\mu}(t)$, for $\mu>\frac{2}{a}, \mu$ integer, the following real function

$$
\rho_{\mu}(t)= \begin{cases}1 & \text { if }-a \leq t \leq-\frac{2}{\mu} \\ 0 & \text { if } t \leq-b \text { and } t \geq-\frac{1}{\mu} \\ \frac{t+b}{b-a} & \text { if }-b<t<-a \\ -(\mu t+1) & \text { if }-\frac{2}{\mu}<t<-\frac{1}{\mu} .\end{cases}
$$

Moreover set $\left\{g_{s}(t)\right\}$ the sequence of symmetric regularizing functions such that

$$
\begin{aligned}
g_{s}(t) & \in C_{0}^{\infty}(\mathbb{R}), \quad g_{s}(t) \geq 0, \quad g_{s}(t)=g_{s}(-t), \\
\operatorname{supp} g_{s} & \subset \quad\left[-\frac{1}{s}, \frac{1}{s}\right], \quad \int_{\mathbb{R}} g_{s}(t) \mathrm{d} t=1 .
\end{aligned}
$$


Let $i$ be a positive integer, $i \leq n$, and $h$ a real number such that $|h|<\frac{\sigma}{2}$. For every $\mu>\frac{2}{a}$ and for every $s>\max \left\{\mu, \frac{1}{T-b}\right\}$, let us define the following "test function"

$$
\varphi(X)=\tau_{i,-h}\left\{\psi^{2 m} \rho_{\mu}\left[\left(\rho_{\mu} \tau_{i, h} u\right) * g_{s}\right]\right\}, \quad \forall X=(x, t) \in Q .
$$

Substituting in (2.2) the above defined function $\phi$, we have

$$
\begin{aligned}
& \int_{Q} \sum_{|\alpha|=m}\left(\tau_{i, h} a^{\alpha}(X, D u) \mid D^{\alpha}\left(\psi^{2 m} \rho_{\mu}\left[\left(\rho_{\mu} \tau_{i, h} u\right) * g_{s}\right]\right)\right) \mathrm{d} X \\
& \quad=\int_{Q}\left(\tau_{i, h} u \mid \psi^{2 m}\left\{\rho_{\mu}\left[\left(\rho_{\mu} \tau_{i, h} u\right) * g_{s}\right]\right\}^{\prime}\right) \mathrm{d} X- \\
& -\sum_{|\alpha|<m} \int_{Q}\left(a^{\alpha}(X, D u) \mid \tau_{i,-h} D^{\alpha}\left\{\psi^{2 m} \rho_{\mu}\left[\left(\rho_{\mu} \tau_{i, h} u\right) * g_{s}\right]\right\}\right) \mathrm{d} X .
\end{aligned}
$$

For every $\alpha:|\alpha|=m$ and a. e. $X=(x, t) \in Q$, we have

$$
\begin{aligned}
& \tau_{i, h} a^{\alpha}(X, D u(X))=a^{\alpha}\left(x+h e^{i}, t, D u\left(x+h e^{i}, t\right)\right)-a^{\alpha}(X, D u(X)) \\
& =\int_{0}^{1} \frac{d}{d \eta} a^{\alpha}\left(x+\eta h e^{i}, t, D u(X)+\eta \tau_{i, h} D u(X)\right) d \eta \\
& =h \int_{0}^{1} \frac{\partial}{\partial x_{i}} a^{\alpha}\left(x+\eta h e^{i}, t, D u(X)+\eta \tau_{i, h} D u(X)\right) d \eta \\
& +\sum_{|\beta| \leq m} \sum_{k=1}^{N}\left(\tau_{i, h} D^{\beta} u_{k}(X)\right) \int_{0}^{1} \frac{\partial}{\partial p_{k}^{\beta}} a^{\alpha}\left(x+\eta h e^{i}, t, D u(X)+\eta \tau_{i, h} D u(X)\right) d \eta \\
& =h \frac{\partial a^{\alpha}}{\partial x_{i}}+\sum_{|\beta| \leq m} \sum_{k=1}^{N}\left(\tau_{i, h} D^{\beta} u_{k}(X)\right) \frac{\partial a^{\alpha}}{\partial p_{k}^{\beta}},
\end{aligned}
$$

where, if $b=b(X, p)$, for simplicity of notation, we set

$$
\tilde{b}(X)=\int_{0}^{1} b\left(x+\eta h e^{i}, t, D u(X)+\eta \tau_{i, h} D u(X)\right) d \eta .
$$

Then, equality (3.18) becomes

$$
\begin{aligned}
& \int_{Q} \sum_{|\alpha|=m}\left(h \frac{\widetilde{\partial a^{\alpha}}}{\partial x_{i}}+\sum_{|\beta| \leq m} \sum_{k=1}^{N}\left(\tau_{i, h} D^{\beta} u_{k}(X)\right) \frac{\widetilde{\partial a^{\alpha}}}{\partial p_{k}^{\beta}} \mid D^{\alpha}\left(\psi^{2 m} \rho_{\mu}\left[\left(\rho_{\mu} \tau_{i, h} u\right) * g_{s}\right]\right)\right) \mathrm{d} X \\
= & \int_{Q} \psi^{2 m} \rho^{\prime} \mu\left(\tau_{i, h} u \mid\left(\rho_{\mu} \tau_{i, h} u\right) * g_{s}\right) \mathrm{d} X+\int_{Q}\left(\tau_{i, h} u \mid \psi^{2 m} \rho_{\mu}\left[\left(\rho_{\mu} \tau_{i, h} u\right) * g_{s}\right]^{\prime}\right) \mathrm{d} X- \\
& \sum_{|\alpha|<m} \int_{Q}\left(a^{\alpha}(X, D u) \mid \tau_{i,-h} D^{\alpha}\left\{\psi^{2 m} \rho_{\mu}\left[\left(\rho_{\mu} \tau_{i, h} u\right) * g_{s}\right]\right\}\right) \mathrm{d} X .
\end{aligned}
$$

Taking into account, for $\alpha:|\alpha|=m$, that

$$
D^{\alpha}\left(\psi^{2 m} \rho_{\mu}\left[\left(\rho_{\mu} \tau_{i, h} u\right) * g_{s}\right]\right)=\psi^{2 m} \rho_{\mu}\left[\left(\rho_{\mu} \tau_{i, h} D^{\alpha} u\right) * g_{s}\right]+\psi^{m} \rho_{\mu} \sum_{\gamma<\alpha} c_{\alpha \gamma}(\psi)\left[\left(\rho_{\mu} \tau_{i, h} D^{\gamma} u\right) * g_{s}\right]
$$


where

$$
\left|c_{\alpha \gamma}(\psi)\right| \leq \frac{c(m, n)}{\sigma^{m-|\gamma|}}
$$

we obtain

$$
\begin{aligned}
& \int_{Q} \psi^{2 m} \rho_{\mu} \sum_{|\alpha|=|\beta|=m} \sum_{k=1}^{N}\left(\left(\tau_{i, h} D^{\beta} u_{k}(X)\right) \frac{\widetilde{\partial a^{\alpha}}}{\partial p_{k}^{\beta}} \mid\left(\rho_{\mu} \tau_{i, h} D^{\alpha} u\right) * g_{s}\right) \mathrm{d} X \\
& =-\sum_{|\alpha|=|\beta|=m} \sum_{\gamma<\alpha} \sum_{k=1}^{N} \int_{Q}\left(\left(\tau_{i, h} D^{\beta} u_{k}(X)\right) \frac{\widetilde{\partial a^{\alpha}}}{\partial p_{k}^{\beta}} \mid \psi^{m} \rho_{\mu} c_{\alpha \gamma}(\psi)\left[\left(\rho_{\mu} \tau_{i, h} D^{\gamma} u\right) * g_{s}\right]\right) \mathrm{d} X \\
& -\sum_{|\alpha|=m|\beta|<m} \sum_{k=1} \int_{Q}^{N}\left(\left(\tau_{i, h} D^{\beta} u_{k}(X)\right) \frac{\widetilde{\partial \sigma^{\alpha}}}{\partial p_{k}^{\beta}} \mid D^{\alpha}\left(\psi^{2 m} \rho_{\mu}\left[\left(\rho_{\mu} \tau_{i, h} u\right) * g_{s}\right]\right)\right) \mathrm{d} X \\
& -h \sum_{|\alpha|=m} \int_{Q}\left(\frac{\partial a^{\alpha}}{\partial x_{i}} \mid D^{\alpha}\left(\psi^{2 m} \rho_{\mu}\left[\left(\rho_{\mu} \tau_{i, h} u\right) * g_{s}\right]\right)\right) \mathrm{d} X+\int_{Q} \psi^{2 m} \rho^{\prime} \mu\left(\tau_{i, h} u \mid\left(\rho_{\mu} \tau_{i, h} u\right) * g_{s}\right) \mathrm{d} X \\
& +\int_{Q}\left(\tau_{i, h} u \mid \psi^{2 m} \rho_{\mu}\left[\left(\rho_{\mu} \tau_{i, h} u\right) * g^{\prime} s\right]\right) \mathrm{d} X-\sum_{|\alpha|<m} \int_{Q}\left(a^{\alpha}(X, D u) \mid \tau_{i,-h} D^{\alpha}\left\{\psi^{2 m} \rho_{\mu}\left[\left(\rho_{\mu} \tau_{i, h} u\right) * g_{s}\right]\right\}\right) \mathrm{d} X .
\end{aligned}
$$

For $s \rightarrow+\infty$, using ellipticity condition (3.6), symmetry hypothesis, convolution property of $g_{s}$ and that

$$
\lim _{s \rightarrow+\infty} \int_{Q}\left(\tau_{i, h} u \mid \psi^{2 m} \rho_{\mu}\left[\left(\rho_{\mu} \tau_{i, h} u\right) * g^{\prime} s\right]\right) \mathrm{d} X=0,
$$

we have

$$
\begin{aligned}
& v \int_{-b}^{-\frac{1}{\mu}} \mathrm{d} t \int_{B(2 \sigma)} \psi^{2 m} \rho_{\mu}^{2}\left\|\tau_{i, h} D^{\prime \prime} u\right\|^{2} \mathrm{~d} x=v \int_{-b}^{-\frac{1}{\mu}} \mathrm{d} t \int_{B(2 \sigma)} \psi^{2 m} \rho_{\mu}^{2} \sum_{|\alpha|=m}\left\|\tau_{i, h} D^{\alpha} u\right\|^{2} \mathrm{~d} x \\
& \leq \int_{Q} \psi^{2 m} \rho_{\mu} \sum_{|\alpha|=|\beta|=m} \sum_{k=1}^{N}\left(\left(\tau_{i, h} D^{\beta} u_{k}(X)\right) \frac{\widetilde{\partial a^{\alpha}}}{\partial p_{k}^{\beta}} \mid \rho_{\mu} \tau_{i, h} D^{\alpha} u\right) \mathrm{d} X \\
& \leq A+B+C+D+E,
\end{aligned}
$$

where

$$
\begin{aligned}
& A=-\sum_{|\alpha|=|\beta|=m} \sum_{\gamma<\alpha} \sum_{k=1}^{N} \int_{Q} c_{\alpha \gamma}(\psi) \psi^{m} \rho_{\mu}^{2}\left(\left(\tau_{i, h} D^{\beta} u_{k}(X)\right) \frac{\widetilde{\partial a^{\alpha}}}{\partial p_{k}^{\beta}} \mid\left(\tau_{i, h} D^{\gamma} u\right)(X)\right) \mathrm{d} X, \\
& B=-\sum_{|\alpha|=m|\beta|<m} \sum_{k=1}^{N} \int_{Q}\left(\left(\tau_{i, h} D^{\beta} u_{k}(X)\right) \frac{\widetilde{\partial a^{\alpha}}}{\partial p_{k}^{\beta}} \mid D^{\alpha}\left(\psi^{2 m} \rho_{\mu}^{2} \tau_{i, h} u(X)\right)\right) \mathrm{d} X, \\
& \left.C=-h \sum_{|\alpha|=m} \int_{Q}\left(\frac{\widetilde{\partial a^{\alpha}}}{\partial x_{i}} \mid D^{\alpha}\left(\psi^{2 m} \rho_{\mu}^{2} \tau_{i, h} u\right)(X)\right)\right) \mathrm{d} X, \\
& D=\int_{Q} \psi^{2 m} \rho^{\prime} \mu \rho_{\mu}\left\|\tau_{i, h} u\right\|^{2} \mathrm{~d} X,
\end{aligned}
$$




$$
E=-\sum_{|\alpha|<m} \int_{Q}\left(a^{\alpha}(X, D u) \mid \tau_{i,-h} D^{\alpha}\left(\psi^{2 m} \rho_{\mu}^{2} \tau_{i, h} u\right)\right) \mathrm{d} X .
$$

We observe that, for every $\varepsilon>0$, we have

$$
|A| \leq \varepsilon \int_{-b}^{-\frac{1}{\mu}} \mathrm{d} t \int_{B(2 \sigma)} \psi^{2 m} \rho_{\mu}^{2}\left\|\tau_{i, h} D^{\prime \prime} u\right\|^{2} \mathrm{~d} x+c(K, \sigma, m, n, \varepsilon) h^{2} \int_{-b}^{-\frac{1}{\mu}} \mathrm{d} t \int_{B(3 \sigma)}\left(1+\left\|D^{\prime \prime} u\right\|^{2}\right) \mathrm{d} x .
$$

The term $B$ can be estimated, for every $\varepsilon>0$, as follows

$$
\begin{aligned}
|B| \leq\left\{\varepsilon+c(K, U, m, n)\left(|h|^{\lambda}+|h|^{2 \lambda}\right)\right\} \int_{-b}^{-\frac{1}{\mu}} \mathrm{d} t \int_{B(2 \sigma)} \psi^{2 m} \rho_{\mu}^{2}\left\|\tau_{i, h} D^{\prime \prime} u\right\|^{2} \mathrm{~d} x \\
+c(K, \sigma, m, n, \varepsilon) h^{2} \int_{-b}^{-\frac{1}{\mu}} \mathrm{d} t \int_{B(3 \sigma)} \|\left. D^{\prime \prime} u\right|^{2} \mathrm{~d} x \\
+c(K, m, n, \varepsilon) \int_{-b}^{-\frac{1}{\mu}} \mathrm{d} t \int_{B(2 \sigma)} \psi^{2 m} \rho_{\mu}^{2}\left\|\tau_{i, h} D^{\prime} u\right\|^{2}\left\|D^{\prime \prime} u\right\|^{2} \mathrm{~d} x .
\end{aligned}
$$

Let us consider the term $C$, for every $\varepsilon>0$, we have

$$
\begin{aligned}
|C| \leq\left\{\varepsilon+c(K, m, n)\left(h^{2}+|h|\right)\right\} \int_{-b}^{-\frac{1}{\mu}} \mathrm{d} t \int_{B(2 \sigma)} \psi^{2 m} \rho_{\mu}^{2}\left\|\tau_{i, h} D^{\prime \prime} u\right\|^{2} \mathrm{~d} x \\
+c(K, \sigma, m, n, \varepsilon) h^{2} \int_{-b}^{-\frac{1}{\mu}} \mathrm{d} t \int_{B(3 \sigma)}\left(1+\left\|D^{\prime \prime} u\right\|^{2}\right) \mathrm{d} x .
\end{aligned}
$$

To estimate the term $D$, we firstly observe that

$$
\left(\rho^{\prime} \mu \rho_{\mu}\right)(t) \begin{cases}=0 & \text { if } t \leq-b,-a \leq t \leq-\frac{2}{\mu}, t \geq-\frac{1}{\mu} \\ \leq \frac{1}{b-a} & \text { if }-b \leq t \leq-a \\ \leq 0 & \text { if }-\frac{2}{\mu} \leq t \leq-\frac{1}{\mu}\end{cases}
$$

then, using Theorem 2.2, we obtain

$$
\begin{aligned}
& D=\int_{Q} \psi^{2 m} \rho^{\prime} \mu \rho_{\mu}\left\|\tau_{i, h} u\right\|^{2} \mathrm{~d} X \\
& =\int_{-b}^{-a} \mathrm{~d} t \int_{B_{(2 \sigma)}} \psi^{2 m} \rho^{\prime} \mu \rho_{\mu}\left\|\tau_{i, h} u\right\|^{2} \mathrm{~d} x+\int_{-\frac{2}{\mu}}^{-\frac{1}{\mu}} \mathrm{d} t \int_{B_{(2 \sigma)}} \psi^{2 m} \rho^{\prime} \mu \rho_{\mu}\left\|\tau_{i, h} u\right\|^{2} \mathrm{~d} x \\
& \quad \leq \frac{1}{b-, a} \int_{-b}^{-a} \mathrm{~d} t \int_{B_{(2 \sigma)}}\left\|\tau_{i, h} u\right\|^{2} \mathrm{~d} x \leq \frac{h^{2}}{b-a} \int_{-b}^{-a} \mathrm{~d} t \int_{B_{(3 \sigma)}}\left\|D_{i} u\right\|^{2} \mathrm{~d} x .
\end{aligned}
$$


Finally, using (3.4) condition, the term $E$ can be expressed as follows

$$
|E| \leq c(K, m, n) \sum_{|\alpha|<m} \int_{-b}^{-\frac{1}{\mu}} \rho_{\mu}^{2} \mathrm{~d} t \int_{B\left(\frac{5}{2} \sigma\right)}\left(\left|f^{\alpha}\right|+\|\left. D^{\prime \prime} u\right|^{2}\right)\left\|\tau_{i,-h} D^{\alpha}\left(\psi^{2 m} \tau_{i, h} u\right)\right\| \mathrm{d} x .
$$

Then, from (3.19) estimating the terms $A, B, C, D$, and $E$, for every $\varepsilon>0$, we have

$$
\begin{aligned}
& \nu \int_{-b^{*}}^{-\frac{1}{\mu}} \mathrm{d} t \int_{B(2 \sigma)} \psi^{2 m} \rho_{\mu}^{2}\left\|\tau_{i, h} \mathrm{D}^{\prime \prime} u\right\|^{2} \mathrm{~d} x \\
& \leq\left\{3 \varepsilon+c(K, U, m, n)\left(|h|+h^{2}+|h|^{\lambda}+|h|^{2 \lambda}\right)\right\} \int_{-b^{*}}^{-\frac{1}{\mu}} \mathrm{d} t \int_{B(2 \sigma)} \psi^{2 m} \rho_{\mu}^{2}\left\|\tau_{i, h} D^{\prime \prime} u\right\|^{2} \mathrm{~d} x \\
& +c(K, \sigma, a, b, m, n, \varepsilon) h^{2} \int_{-b^{*}}^{-\frac{1}{\mu}} \mathrm{d} t \int_{B(3 \sigma)}\left(1+\left\|D^{\prime \prime} u\right\|^{2}\right) \mathrm{d} x+c(\sigma, a, b, n,) K h^{2} \\
& \quad-\frac{1}{\mu} \int_{-b^{*}} \mathrm{~d} t \int_{B(2 \sigma)} \psi^{2 m} \rho_{\mu}^{2}\left\|\tau_{i, h} D^{\prime} u\right\|^{2}\left\|D^{\prime \prime} u\right\|^{2} \mathrm{~d} x \\
& +c\left(K, \sigma, m, n, \frac{1}{\mu}\right. \\
& +c(K, m, n) \sum_{|\alpha|<m} \int_{-b^{*}}^{-} \rho_{\mu}^{2} \mathrm{~d} t \int_{B}\left(\mid \frac{5}{2} \sigma\right)
\end{aligned}
$$

We observe that the function

$$
h \rightarrow c(K, U, \sigma, m, n)\left(|h|+h^{2}+|h|^{\lambda}+|h|^{2 \lambda}\right)
$$

is continuous in the origin, then $\exists h_{0}(v, K, U, \lambda, \sigma, m, n), 0<h_{0}<\min \left\{1, \frac{\sigma}{2}\right\}$, such that for every $|h|<h_{0}$, we have

$$
c(K, U, \sigma, m, n)\left(|h|+h^{2}+|h|^{\lambda}+|h|^{2 \lambda}\right)<\frac{v}{4} .
$$

For each integer $i=1, \ldots, n$, for $\varepsilon=\frac{v}{12}$ and every $h$ such that $|h|<h_{0}(<1)$, it follows

$$
\begin{aligned}
& \frac{v}{2} \int_{-b^{*}}^{-\frac{1}{\mu}} \mathrm{d} t \int_{B(2 \sigma)} \psi^{2 m} \rho_{\mu}^{2}\left\|\tau_{i, h} D^{\prime \prime} u\right\|^{2} \mathrm{~d} x \\
& \leq c(v, K, \sigma, a, b, m, n)|h|^{2} \int_{-b^{*}}^{-\frac{1}{\mu}} \mathrm{d} t \int_{B(3 \sigma)}\left(1+\|\left. D^{\prime \prime} u\right|^{2}\right) \mathrm{d} x \\
& +c(v, K, \sigma, m, n) \int_{-b^{*}}^{-\frac{1}{\mu}} \mathrm{d} t \int_{B(2 \sigma)} \psi^{2 m} \rho_{\mu}^{2}\left\|\tau_{i, h} D^{\prime} u\right\|^{2}\left\|D^{\prime \prime} u\right\|^{2} \mathrm{~d} x \\
& +c(K, m, n) \sum_{|\alpha|<m} \int_{-b^{*}}^{-\frac{1}{\mu}} \rho_{\mu}^{2} \mathrm{~d} t \int_{B\left(\frac{5}{2} \sigma\right)}\left(\left|f^{\alpha}\right|+\left\|D^{\prime \prime} u\right\|^{2}\right)\left\|\tau_{i,-h} D^{\alpha}\left(\psi^{2 m} \tau_{i, h} u\right)\right\| \mathrm{d} x .
\end{aligned}
$$


Let us focus our attention on the last term, taking into account that from (3.12), for a. e. $t \in\left(-b^{*}, 0\right)$, we have

$$
u(\cdot, t) \in H^{m, 4}\left(B\left(\frac{5}{2} \sigma\right), \mathbb{R}^{N}\right)
$$

then using Hölder and Young inequalities, for every $\alpha$ such that $|\alpha|<m$, for every $\varepsilon>0$, it follows

$$
\begin{aligned}
& \int_{B\left(\frac{5}{2} \sigma\right)}\left(\left|f^{\alpha}\right|+\left\|D^{\prime \prime} u\right\|^{2}\right)\left\|\tau_{i,-h} D^{\alpha}\left(\psi^{2 m} \tau_{i, h} u\right)\right\| \mathrm{d} x \\
\leq & \left(\int_{B(3 \sigma)}|h|^{-2}\left\|\tau_{i,-h} D^{\alpha}\left(\psi^{2 m} \tau_{i, h} u\right)\right\|^{2} \mathrm{~d} x\right)^{\frac{1}{2}}\left(\int_{B\left(\frac{5}{2} \sigma\right)} h^{2}\left(\left|f^{\alpha}\right|+\left\|D^{\prime \prime} u\right\|^{2}\right)^{2} \mathrm{~d} x\right)^{\frac{1}{2}} \\
\leq & \frac{\varepsilon}{2}|h|^{-2} \int_{B(3 \sigma)}\left\|\tau_{i,-h} D^{\alpha}\left(\psi^{2 m} \tau_{i, h} u\right)\right\|^{2} \mathrm{~d} x+c(\varepsilon) h^{2} \int_{B\left(\frac{5}{2} \sigma\right)}\left(\left|f^{\alpha}\right|^{2}+\left\|D^{\prime \prime} u\right\|^{4}\right) \mathrm{d} x .
\end{aligned}
$$

Furthermore, for every $\alpha$ such that $|\alpha|<m$, from Theorem 2.2 for every $h \in \mathbb{R}$ with $|h|<h_{0}$ and for every $\varepsilon>0$, we have

$$
\begin{aligned}
& \frac{\varepsilon}{2}|h|^{-2} \int_{B(3 \sigma)}\left\|\tau_{i,-h} D^{\alpha}\left(\psi^{2 m} \tau_{i, h} u\right)\right\|^{2} \mathrm{~d} x \leq \frac{\varepsilon}{2} \int_{B\left(\frac{7}{2} \sigma\right)}\left\|D^{\prime \prime}\left(\psi^{2 m} \tau_{i, h} u\right)\right\|^{2} \mathrm{~d} x \\
& \quad \leq \varepsilon \int_{B(2 \sigma)} \psi^{2 m}\left\|\tau_{i, h} D^{\prime \prime} u\right\|^{2} \mathrm{~d} x+c(\sigma, \varepsilon) \int_{B(2 \sigma)}\left\|\tau_{i, h} D^{\prime} u\right\|^{2} \mathrm{~d} x \\
& \quad \leq \varepsilon \int_{B(2 \sigma)} \psi^{2 m}\left\|\tau_{i, h} D^{\prime \prime} u\right\|^{2} \mathrm{~d} x+c(\sigma, \varepsilon) h^{2} \int_{B(3 \sigma)}\left\|D^{\prime \prime} u\right\|^{2} \mathrm{~d} x
\end{aligned}
$$

the last inequality follows, as before, applying Theorem 2.2 for $p=2$.

Let us now choose $\varepsilon=\frac{v}{4 c(K, m, n)}$, it ensures

$$
\begin{array}{r}
\int_{B\left(\frac{5}{2} \sigma\right)}\left(\left|f^{\alpha}\right|+|| D^{\prime \prime} u \|^{2}\right)\left\|\tau_{i,-h} D^{\alpha}\left(\psi^{2 m} \tau_{i, h} u\right)\right\| \mathrm{d} x \\
\leq \frac{v}{4 c(K, m, n)} \int_{B(2 \sigma)} \psi^{2 m}|| \tau_{i, h} D^{\prime \prime} u \|^{2} \mathrm{~d} x+c(v, K, \sigma, m, n) h^{2}\left\{\int_{B(3 \sigma)}\left|f^{\alpha}\right|^{2} \mathrm{~d} x+|u|_{m, B(3 \sigma)}^{2}+|u|_{m, 4, B\left(\frac{5}{2} \sigma\right)}^{4}\right\} .
\end{array}
$$

Multiplying each term for $\rho_{\mu}^{2}$ and integrating respect to $\left(-b^{*},-\frac{1}{\mu}\right)$ and applying (3.13), we achieve 


$$
\begin{aligned}
& \int_{-b^{*}}^{-\frac{1}{\mu}} \rho_{\mu}^{2} \mathrm{~d} t \int_{B\left(\frac{5}{2} \sigma\right)}\left(\left|f^{\alpha}\right|+\left\|D^{\prime \prime} u\right\|^{2}\right)\left\|\tau_{i,-h} D^{\alpha}\left(\psi^{2 m} \tau_{i, h} u\right)\right\| \mathrm{d} x \\
& \leq \frac{v}{4 c(K, m, n)} \int_{-b^{*}}^{-\frac{1}{\mu}} \rho_{\mu}^{2} \mathrm{~d} t \int_{B(2 \sigma)} \psi^{2 m}\left\|\tau_{i, h} D^{\prime \prime} u\right\|^{2} \mathrm{~d} x \\
& +c(\nu, K, U, \lambda, \sigma, a, b, m, n) h^{2}\left\{1+\sum_{|\alpha|<m}\left(\int_{-b}^{0}\left\|f^{\alpha} \mid\right\|_{0, B(3 \sigma)} \mathrm{d} t\right)^{\frac{1+\vartheta}{2}}+\int_{-b^{*}}^{-\frac{1}{\mu}}|u|_{m, B(3 \sigma)}^{2} \mathrm{~d} t\right\} .
\end{aligned}
$$

Taking into consideration the last inequality and the properties of the function $\psi$, from (3.31) we deduce

$$
\begin{aligned}
& \int_{-a}^{-\frac{2}{\mu}} \mathrm{d} t \int_{B(\sigma)}\left\|\tau_{i, h} D^{\prime \prime} u\right\|^{2} \mathrm{~d} x \\
& \leq c(v, K, U, \lambda, \sigma, a, b, m, n) h^{2}\left\{1+\sum_{|\alpha|<m}\left(\int_{-b}^{0}\left\|f^{\alpha}\right\|_{0, B(3 \sigma)} \mathrm{d} t\right)^{\frac{1+\vartheta}{2}}+\int_{-b^{*}}^{-\frac{1}{\mu}}|u|_{m, B(3 \sigma)}^{2} \mathrm{~d} t\right\} \\
& +c(v, K, \sigma, m, n) \int_{-b^{*}}^{-\frac{1}{\mu}} \mathrm{d} t \int_{B(2 \sigma)} \psi^{2 m} \rho_{\mu}^{2}\left\|\tau_{i, h} D^{\prime} u\right\|^{2}\left\|D^{\prime \prime} u\right\|^{2} \mathrm{~d} x .
\end{aligned}
$$

From which, passing the limit $\mu \rightarrow \infty$, we get

$$
\begin{aligned}
& \int_{-a}^{0} \mathrm{~d} t \int_{B(\sigma)}\left\|\tau_{i, h} D^{\prime \prime} u\right\|^{2} \mathrm{~d} x \\
& \leq c(\nu, K, \lambda, \sigma, m, n) h^{2}\left\{1+\sum_{|\alpha|<m}\left(\int_{-b}^{0}\left\|f^{\alpha}\right\| \|_{0, B(3 \sigma)} \mathrm{d} t\right)^{\frac{1+\vartheta}{2}}+\int_{-b^{*}}^{0}|u|_{m, B(3 \sigma)}^{2} \mathrm{~d} t\right\} \\
& +c(\nu, K, \sigma, m, n) \int_{-b^{*}}^{0} \mathrm{~d} t \int_{B(2 \sigma)} \psi^{2 m} \rho_{\mu}^{2}\left\|\tau_{i, h} D^{\prime} u\right\|^{2}\left\|D^{\prime \prime} u\right\|^{2} \mathrm{~d} x .
\end{aligned}
$$

Let us now estimate the last term in (3.32). Using Hölder inequality, applying Theorem 2.2 (for $p=4, B\left(\frac{5}{2} \sigma\right)$ instead of $B(\sigma)$ and $t=\frac{4}{5}$ ) and formula (3.13), for every $|h|<$ $h_{0}$, it follows

$$
\begin{gathered}
\int_{B(2 \sigma)}\left\|\tau_{i, h} D^{\prime} u\right\|^{2}\left\|D^{\prime \prime} u\right\|^{2} \mathrm{~d} x \leq\left(\int_{B(2 \sigma)}\left\|\tau_{i, h} D^{\prime} u\right\|^{4} \mathrm{~d} x\right)^{\frac{1}{2}}\left(\int_{B(2 \sigma)}\left\|D^{\prime \prime} u\right\|^{4} \mathrm{~d} x\right)^{\frac{1}{2}} \\
\leq|h|^{2}\left\|D^{\prime \prime} u\right\|_{0,4, B\left(\frac{5}{2} \sigma\right)}^{2}\left\|D^{\prime \prime} u\right\|_{0,4, B(2 \sigma)}^{2} \leq|h|^{2}|u|_{m, 4, B\left(\frac{5}{2} \sigma\right)}^{4} .
\end{gathered}
$$


Integrating in $\left(-b^{*}, 0\right)$, from (3.32), it follows

$$
\begin{aligned}
& \int_{-a}^{0} \mathrm{~d} t \int_{B(\sigma)}\left\|\tau_{i, h} D^{\prime \prime} u\right\|^{2} \mathrm{~d} x \\
& \leq c(\nu, K, U, \lambda, \sigma, a, b, m, n)|h|^{2}\left\{1+\sum_{|\alpha|<m}\left(\int_{-b}^{0}\left\|f^{\alpha}\right\|_{0, B(3 \sigma)} \mathrm{d} t\right)^{\frac{1+\vartheta}{2}}+\int_{-b}^{0}|u|_{m, B(3 \sigma)}^{2} \mathrm{~d} t\right\} .
\end{aligned}
$$

If $h_{0} \leq|h|<\frac{\sigma}{2}$, for every $i=1,2, \ldots, n$ we easily obtain

$$
\begin{aligned}
& \int_{-a}^{0} \mathrm{~d} t \int_{B \sigma)}\left\|\tau_{i, h} D^{\prime \prime} u\right\|^{2} \mathrm{~d} x \leq 4 \int_{-a}^{0} \mathrm{~d} t \int_{B(3 \sigma)}\left\|\left.D^{\prime \prime} u\right|^{2} \mathrm{~d} x \leq 4 \frac{h^{2}}{h_{0}^{2}} \int_{-a}^{0} \mathrm{~d} t \int_{B(3 \sigma)}\right\| D^{\prime \prime} u \|^{2} \mathrm{~d} x \\
\leq & c(v, K, U, \lambda, \sigma, a, b, m, n) h^{2} \int_{-b}^{0}|u|_{m, B(3 \sigma)}^{2} \mathrm{~d} t \\
\leq & c(v, K, U, \lambda, \sigma, a, b, m, n)|h|^{2}\left\{1+\sum_{|\alpha|<m}\left(\int_{-b}^{0}|| f^{\alpha}||_{0, B(3 \sigma)} \mathrm{d} t\right)^{\frac{1+\vartheta}{2}}+\int_{-b}^{0}|u|_{m, B(3 \sigma)}^{2} \mathrm{~d} t\right\} .
\end{aligned}
$$

It is then proved, for every $|h|<\frac{\sigma}{2}$ and every $i \in\{1,2, \ldots, n\}$, that

$$
\begin{aligned}
& \int_{-a}^{0} \mathrm{~d} t \int_{B(\sigma)}\left\|\tau_{i, h} D^{\prime \prime} u\right\|^{2} \mathrm{~d} x \\
& \leq c(v, K, U, \lambda, \sigma, a, b, m, n)|h|^{2}\left\{1+\sum_{|\alpha|<m}\left(\int_{-b}^{0}\left\|f^{\alpha}\right\|_{0, B(3 \sigma)} \mathrm{d} t\right)^{\frac{1+\vartheta}{2}}+\int_{-b}^{0}|u|_{m, B(3 \sigma)}^{2} \mathrm{~d} t\right\},
\end{aligned}
$$

applying Theorem 2.1, it follows

$$
u \in L^{2}\left(-a, 0, H^{m+1}\left(B(\sigma), \mathbb{R}^{N}\right)\right)
$$

and

$$
\begin{aligned}
& \int_{-a}^{0}|u|_{m+1, B(\sigma)}^{2} \mathrm{~d} t \\
& \leq c(v, K, U, \lambda, \sigma, a, b, m, n)\left\{1+\sum_{|\alpha|<m}\left(\int_{-b}^{0}|| f^{\alpha} \|_{0, B(3 \sigma)} \mathrm{d} t\right)^{\frac{1+\vartheta}{2}}+\int_{-b}^{0}|u|_{m, B(3 \sigma)}^{2} \mathrm{~d} t\right\} .
\end{aligned}
$$

Finally we have to prove that $u \in H^{1}\left(-a, 0, L^{2}\left(B(\sigma), \mathbb{R}^{N}\right)\right)$ and inequality (3.15). 
From inequality (3.8), we have

$$
\begin{aligned}
& \int_{-a}^{0} \mathrm{~d} t \int_{B(\sigma)}\left\|D^{\prime \prime} u\right\|^{4} \mathrm{~d} x \\
& \leq c(v, K, U, \lambda, \sigma, a, b, m, n)\left\{1+\sum_{|\alpha|<m}\left(\int_{-b}^{0}|| f^{\alpha} \|_{0, B(3 \sigma)} \mathrm{d} t\right)^{\frac{1+\vartheta}{2}}+\int_{-b}^{0}|u|_{m, B(3 \sigma)}^{2} \mathrm{~d} t\right\}
\end{aligned}
$$

then we have

$$
D^{\prime \prime} u \in L^{4}\left(B(\sigma) \times(-a, 0), \mathcal{R}^{\prime \prime}\right) .
$$

Moreover, bearing in mind that, for $|\alpha|<m, a^{\alpha}(X, p)$ satisfies (3.4), and for $|\alpha|=m$, $a^{\alpha}(X, p)$ satisfies (3.5), we have

$$
D^{\alpha} a^{\alpha}(X, p) \in L^{2}\left(B(\sigma) \times(-a, 0), R^{N}\right) \quad \forall \alpha:|\alpha| \leq m
$$

Recalling the definition of weak solution, for every $\phi \in C_{0}^{\infty}\left(Q, \mathbb{R}^{N}\right)$, proceeding as in [22], we have

$$
\int_{-a}^{0} \mathrm{~d} t \int_{B(\sigma)}\left(u \mid \frac{\partial \varphi}{\partial t}\right) \mathrm{d} x=\sum_{|\alpha| \leq m} \int_{-a}^{0} \mathrm{~d} t \int_{B(\sigma)}\left(D^{\alpha} a^{\alpha}(X, D u) \mid \varphi\right) \mathrm{d} x,
$$

and, bearing in mind (3.37), we obtain that

$$
\exists \frac{\partial u}{\partial t} \in L^{2}\left(B(\sigma) \times(-a, 0), \mathbb{R}^{N}\right) .
$$

From (3.4), (3.5) and (3.38), we get

$$
\begin{aligned}
& \int_{-a}^{0} \mathrm{~d} t \int_{B(\sigma)}\left\|\frac{\partial u}{\partial t}\right\|^{2} \mathrm{~d} x \\
& \leq c(v, K, U, \lambda, \sigma, a, b, m, n)\left\{1+\sum_{|\alpha|<m}\left(\int_{-b}^{0}\left\|f^{\alpha}\right\|_{0, B(3 \sigma)} \mathrm{d} t\right)^{2}+\int_{-b}^{0}|u|_{m, B(3 \sigma)}^{2} \mathrm{~d} t\right\} .
\end{aligned}
$$

The last inequality and (3.34) allows us to conclude the proof.

\section{Authors' contributions}

The contributions of all the authors are equals. All the authors read and approved the final manuscript.

\section{Competing interests}

The authors declare that they have no competing interests.

Received: 13 January 2011 Accepted: 31 August 2011 Published: 31 August 2011

References

1. Marino, M, Maugeri, A: Generalized Gagliardo-Nirenberg estimates and differentiability of the solutions to monotone nonlinear parabolic systems. J Global Optim. 40, 185-196 (2008). doi:10.1007/s10898-007-9184-7

2. Ragusa, MA: Local Hölder regularity for solutions of elliptic systems. Duke Math J. 113(2), 385-397 (2002). doi:10.1215/ S0012-7094-02-11327-1

3. Ragusa, MA: Parabolic systems with non-continuous coefficients. Dynamical systems and differential equations. Discrete Contin Dyn Syst (suppl). 727-733 (2003) 
4. Floridia, G, Ragusa, MA: Differentiability and partial Holder continuity of solutions of solutions of nonlinear elliptic systems. To appear in J Convex Anal. 19(1) (2012)

5. Campanato, S, Cannarsa, P: Differentiability and partial Hölder continuity of the solutions of nonlinear elliptic systems of order $2 m$ with quadratic growth. Ann Scuola Norm Sup Pisa CI Sci (4). 8(2), 285-309 (1981)

6. Triebel, H: Interpolation theory, function spaces, differential operators. pp. 528. North-Holland Mathematical Library, 18, North-Holland Publishing Co., Amsterdam-New York (1978)

7. Triebel, H: Theory of Function Spaces. Monographs in Mathematics, 78. pp. 284. Birkhäuser Verlag, Basel (1983)

8. Fattorusso, L: Sulla differenziabilitá delle soluzioni di sistemi parabolici non lineari del secondo ordine ad andamento quadratico. Boll Un Mat Ital B (7). 1(3), 741-764 (1987)

9. Marino, M, Maugeri, A: Differentiability of weak solutions of nonlinear parabolic systems with quadratic growth. Matematiche Catania. 50, 361-377 (1995)

10. Marino, M: Equazioni differenziali e...altro. Boll Accademia Gioenia Catania. 42(371), 1-15 (2010)

11. Fattorusso, L, Marino, M: Interior differentiability results for nonlinear variational parabolic systems with nonlinearity $1<$ $q<2$. Med J Math. , 8: 97-112 (2011)

12. Naumann, J: On the interior differentiability of weak solutions of parabolic systems with quadratic growth nonlinearities. Rend Sem Mat Univ Padova. 83, 55-70 (1990)

13. Naumann, J, Wolf, J: Interior differentiability of weak solutions to parabolic systems with quadratic growth. Rend Sem Mat Univ Padova. 98, 253-272 (1997)

14. Campanato, S: On the nonlinear parabolic systems in divergence form. Hölder continuity and partial Hölder continuity of the solutions. Ann Mat Pura Appl. 137(4), 83-122 (1984)

15. Adams, RA: Sobolev Spaces. In Pure and Applied Mathematics, vol. 65, pp. xviii+268.Academic Press, New York (1975)

16. Kufner, A, John, O, Fučik, S: Function Spaces. Monographs and Textbooks on Mechanics of Solids and Fluids; Mechanics: Analysis. pp. xv+454. Noordhoff International Publishing, Leyden; Academia, Prague (1977)

17. John, F, Nirenberg, L: On functions of bounded mean oscillation. Comm Pure Appl Math. 14, 415-426 (1961). doi:10.1002/cpa.3160140317

18. Nirenberg, L: An extended interpolation inequality. Ann Scuola Norm Sup Pisa. 20(3), 733-737 (1966)

19. Campanato, S: Sistemi ellittici in forma divergenza. Regolaritá all'interno. Quaderni, Scuola Normale Superiore Pisa, Pisa. 187 (1980)

20. Miranda, C: Su alcuni teoremi di inclusione. Annales Polon Math. 16, 305-315 (1965)

21. Fattorusso, L: Sulla differenziabilitá delle soluzioni deboli di sistemi parabolci non lineari di ordine $2 m$ ad andamento quadratico. Matematiche Catania. , 40: 199-215 (1985)

22. Marino, M, Maugeri, A: A remark on the note: partial Hölder continuity of the spatial derivatives of the solutions to nonlinear parabolic systems with quadratic growth. Rend Sem Mat Univ Padova. 95, $23-28$ (1996)

doi:10.1186/1029-242X-2011-42

Cite this article as: Floridia and Ragusa: Interpolation inequalities for weak solutions of nonlinear parabolic systems. Journal of Inequalities and Applications 2011 2011:42.

\section{Submit your manuscript to a SpringerOpen ${ }^{\circ}$ journal and benefit from:}

- Convenient online submission

- Rigorous peer review

- Immediate publication on acceptance

- Open access: articles freely available online

- High visibility within the field

- Retaining the copyright to your article

Submit your next manuscript at $\gg$ springeropen.com 